\title{
Caracterización de grasas para caldos deshidratados
}

\author{
Por R. Correa-Cabrera, A. Capote, M.N. Rodríguez-Ayán y M.A. Grompone* \\ Laboratorio de Grasas y Aceites, Cátedra de Fisicoquímica, Facultad de Química. \\ Avda. Gral. Flores 2124, C.P. 11800, Montevideo, Uruguay.
}

\section{RESUMEN}

\section{Caracterización de grasas para caldos deshidratados.}

Las grasas constituyen un alto porcentaje del peso, de los caldos deshidratados envasados bajo forma de cubitos. Como estos alimentos se almacenan a temperatura ambiente, sus componentes grasos no deben fundir parcialmente durante el verano.

En este trabajo se analizan seis muestras de materias primas grasas, mezclas de ellas ya preparadas por la empresa elaboradora y extractos lipídicos del producto terminado (sopas de gallina y de verduras). Se busca relacionar la naturaleza de las grasas (composición en ácidos grasos) con su estabilidad frente a variaciones de la temperatura (termogramas por DSC). Se concluye que, si bien algunas de las grasas estudiadas son aceptables desde el punto de vista de la vida útil esperada del producto, otras se deberían modificar por hidrogenación o fraccionamiento antes de su uso.

PALABRAS-CLAVE: Caldo deshidratado - Calorimetría diferencial de barrido (DSC) - Estabilidad térmica - Grasa.

\section{SUMMARY}

\section{Dehydrated soup cubes fatty components characterization.}

Fats represent a major part of the weight of dehydrated soup cubes. As these products are stored at room temperature, the fatty components should show no partial melting during the Summer months.

Six fatty raw materials, mixtures of these fats already prepared by the manufacturer and lipidic extracts from the finished cubes (both hen and vegetables soups) are analyzed in this work, trying to relate origin (fatty acid composition) with stability against temperature changes (DSC thermogram). Some of the studied fats are found acceptable according to the expected shelf life of the product, although others should be modified before usage, either by fractioning or by hydrogenation.

KEY-WORDS: Dehydrated soup cube - Differential scanning calorimetry (DSC) - Fat - Thermal stability.

\section{INTRODUCCION}

Los caldos deshidratados, envasados bajo forma de "cubitos", son un alimento ampliamente usado en muchos países. Un porcentaje alto de su peso corresponde a grasas, las cuales no sólo contribuyen de manera importante a las propiedades organolépticas, sino que también le dan forma y resistencia al producto durante el almacenamiento. Los cubos se envuelven en un polilaminado de aluminio y papel y se acondicionan en un estuche de cartón. Se suelen almacenar a temperatura ambiente, sin refrigeración.

Por ello, se debe evitar que, aún durante los días de temperatura alta del verano, sus materiales grasos fundan parcialmente, no sólo para que se mantenga firme su forma paralelepipédica sino también para que no escurra líquido. Esta migración de grasa líquida hacia la superficie del cubito y luego a través de los pliegues de su envoltura es responsable de las manchas oleosas que aparecen en los envases de cartón, impresionando muy desfavorablemente al consumidor. Por otra parte, la gran superficie de exposición al aire de dichas manchas, y las temperaturas altas, hacen que el aceite que las forma se enrancie rápidamente, fenómeno fácilmente percibido por su olor característico.

Se debe considerar, además, que el consumidor prepara el caldo sumergiendo dichos cubitos en agua caliente, por lo que la grasa que contienen deberá estar fundida a la temperatura de su ingesta (la cual varía entre los $50^{\circ}$ y los $70^{\circ} \mathrm{C}$ ).

Debido a todas estas razones, los materiales grasos contenidos en los caldos deshidratados y envasados bajo forma de cubitos deben poseer determinadas propiedades físicas, que el fabricante debe cuidar. Fundamentalmente, deben resistir las variaciones de temperatura ambiente, como las que tienen lugar en el Uruguay entre el verano (más de $30^{\circ} \mathrm{C}$ ) y el invierno (menos de $5^{\circ} \mathrm{C}$ ), sin fundir apreciablemente, y deben estar totalmente líquidos a la temperatura de su ingesta. Por ello es importante la determinación del comportamiento térmico de los materiales grasos empleados, es decir, el cambio en el contenido de fase líquida en función de la temperatura. A su vez, el comportamiento térmico se vincula con la composición en triacilgliceroles, y éstos, en gran medida, con su composición en ácidos grasos (Dubinsky, 1995; Al-Rashood, 1996; Man, 1995; Kapseu, 1994; Zeitoun, 1993).

Generalmente, se emplean como materias primas, además de otros componentes propios del caldo (verduras deshidratadas, carne de gallina deshidratada, extracto de carne, extracto de levadura, especias, proteína vegetal hidrolizada, maltodextrina, harina de trigo, azúcar, caldo concentrado, sal, etc), 
grasas vacunas y/o aceites vegetales parcialmente hidrogenados (soja, maíz, algodón, etc). En este último caso, la hidrogenación suele ser bastante profunda para obtener productos más "duros» que para otros usos (tales como «shortenings" para repostería). En algunos casos también se adicionan algunas grasas especiales como complemento del sabor. Así, en los caldos de gallina, el agregado de grasa de gallina es fundamental desde el punto de vista organoléptico y no por su contribución positiva a las propiedades físicas.

El objetivo general de este trabajo radica en determinar cuáles son las materias primas más adecuadas para fabricar caldos en cubitos, desde el punto de vista de su comportamiento térmico, e inversamente, establecer cuáles son las características que deben poseer las materias primas para ser usadas en este tipo de alimentos. Este estudio viene a llenar un vacio que existe en la bibliografía respecto al tema.

El objetivo específico de este trabajo se centra en la comparación de las materias grasas contenidas en caldos de verdura y de gallina, por lo que contemplará: a) el estudio de las materias grasas utilizadas en la elaboración de caldos deshidratados en cubitos, en cuanto a su perfil térmico y a su composición en ácidos grasos; b) la obtención de información acerca del comportamiento de los cubitos de caldo como tales frente a variaciones importantes de temperatura.

Para cumplir con los objetivos propuestos se estudió, separada y comparativamente, las posibles materias primas empleadas por el fabricante de los caldos de gallina y de verdura, las mezclas por él preparadas y los cubitos de caldo ya elaborados.

\section{PARTE EXPERIMENTAL}

Se estudiaron seis muestras diferentes de grasas usadas como materias primas en la fabricación de diferentes caldos (grasa vacuna refinada, grasa vacuna hidrogenada, aceite vegetal hidrogenado sin indicación de origen, aceite hidrogenado de soja, aceite hidrogenado de maíz y grasa de gallina), dos muestras de mezclas de grasas preparadas por el fabricante para su uso en los caldos de gallina y de verduras, y dos muestras de cubitos de caldo de gallina y de verduras ya elaborados. Todas las muestras fueron proporcionadas por el fabricante de los caldos.

En el caso de los cubitos de caldo, se llevó a cabo una extracción de la porción lipídica por inmersión del producto (fragmentado en trozos pequeños), en éter de petróleo (rango de ebullición: $60^{\circ} \mathrm{C}-80^{\circ} \mathrm{C}$ ), a temperatura ambiente, durante dos horas. El solvente del extracto lipídico obtenido se eliminó en un Rotavapor.

\subsection{Cromatografía de gases (GC)}

La composición en ácidos grasos se determinó por GC de sus ésteres metílicos, de acuerdo con el metodo oficial Ce 2-66 (AOCS, 1993). Se utilizó un cromatografo Hewlett-Packard 5840A con integrador, equipado con columna rellena SP 2330 de la firma Supelco. Las condiciones de operación fueron: temperatura de columna, $185^{\circ} \mathrm{C}$; temperatura del detector (FID), $260^{\circ} \mathrm{C}$; temperatura del inyector, $220^{\circ} \mathrm{C}$. Se usó nitrógeno como gas portador, a $15 \mathrm{~mL} / \mathrm{min}$.

\subsection{Calorimetría diferencial de barrido (DSC)}

Para obtener las curvas de fusión de las muestras se utilizó un calorímetro Shimadzu DSC-50, con procesador y accesorio LTC-50 para bajas temperaturas (como refrigerante se utilizó una mezcla acetona anhídrido carbónico sólido), en las siguientes condiciones: nitrógeno como gas de purga a $50 \mathrm{~mL} / \mathrm{min}$, barrido en el rango de temperaturas de $-30^{\circ} \mathrm{C}$ a $70^{\circ} \mathrm{C}$, velocidad de calentamiento de $5^{\circ} \mathrm{C} / \mathrm{min}$, peso de muestra entre 3 y $5 \mathrm{mg}$. Como software se utilizó el Thermal Analysis System TA-50 WSI de Shimadzu.

A los efectos de determinar las propiedades térmicas, es muy importante el valor de la $T_{\text {onset, }}$ puesto que ella indica la temperatura a la cual comienza la fusión del material graso, y el de la $T_{\text {endset}}$, puesto que ella indica la temperatura a la cual finaliza la fusión. La diferencia entre ambos valores es una indicación del rango de temperaturas que abarca el proceso de fusión. La ubicación de la temperatura correspondiente al máximo del pico ( $T_{\text {pico }}$ ) dentro del rango de fusión, da una indicación aproximada de la temperatura a la cual se formó una cantidad importante de líquido (Garti, 1988).

A partir de los datos de las áreas parciales y del área total del pico correspondiente a la fusión (determinados usando el programa de cálculo numérico del calorímetro), se calculó el porcentaje de sólidos (o su equivalente porcentaje de líquido) a cada una de las temperaturas elegidas durante dicha transición, tal como se menciona en la literatura (Amer, 1985; Fouad, 1990; Borwankar, 1992; Bornaz, 1994; Dimick, 1996).

\subsection{Punto de fusión (PF)}

El PF se determinó por las técnicas de capilar abierto Cc 1-25 y capilar cerrado Cc 3-25 (AOCS, 1993). Se comparan los valores con las $T_{\text {onset, }}$ las $T_{\text {endsot }} y$ las $T_{\text {pico }}$ obtenidas de los termogramas.

El PF medido en capilar cerrado indica la fusión casi completa del material graso ya que se determina por desaparición total de la turbidez (se suele estimar que el contenido de sólidos en ese momento es inferior al $8 \%$ ). EI PF medido a capilar abierto indica una temperatura de ablandamiento de 
la grasa, bajo las condiciones establecidas en su determinación. En consecuencia, el PF en capilar cerrado debería ser ligeramente menor que la $T_{\text {endset }}$ $y$, según la forma del termograma, en muchos casos podría estar próximo a la $T_{\text {pico. }}$ Además la $T_{\text {onset }}$ debería ser muy inferior al PF en capilar abierto, si el termograma presentara un pico de fusión de base ancha.

\section{RESULTADOS Y DISCUSION}

Los resultados obtenidos se agrupan en: 3.1.Materias primas, que incluye las seis grasas usadas en la elaboración, y 3.2.- Caldos, que abarca tanto las mezclas de grasas ya preparadas como los extractos lipídicos de los cubitos de caldo.

\subsection{Materias primas}

En la Tabla I se resumen los valores de las temperaturas $T_{\text {onset, }}, T_{\text {pico }}$ y $T_{\text {endset }}$ obtenidas a partir de los termogramas realizados con cada una de las materias primas (los que, por brevedad, se omiten en este trabajo). La grasa de gallina, el aceite vegetal hidrogenado y el aceite de maíz presentan dos picos sucesivos; se supone que ambos corresponden a fusiones de grupos moleculares con diferentes propiedades térmicas y no a transiciones de formas cristalinas. En consecuencia, se tomó como $T_{\text {onset }}$ la temperatura del comienzo del primer pico, es decir, la temperatura en la que empezaría la primera fusión. El resto de las materias primas presentan un solo pico importante. También se indica en la Tabla I los PF determinados en capilar abierto y en capilar cerrado.

\section{Tabla I}

Puntos de fusión de capilar abierto (PF(ca)) y capilar cerrado (PF(cc)), temperatura "onset», temperatura de pico y temperatura "endset" del termograma para las materias primas grasas (datos en ${ }^{\circ} \mathrm{C}$ )

\begin{tabular}{lccccc}
\hline \multicolumn{1}{c}{ Muestra } & PF(ca) & PF(cc) & $T_{\text {onset }}$ & $T_{\text {pico }}$ & $T_{\text {endset }}$ \\
\hline Grasa vacuna refinada & 49.2 & 49.6 & 26 & 51.5 & 60 \\
Grasa vacuna hidrogenada & 51.2 & 52.0 & 26 & 52.2 & 60 \\
Aceite vegetal hidrogenado & 38.8 & 39.0 & 15 & 37.2 & 45 \\
Aceite soya hidrogenado & 46.0 & 46.6 & 5 & 47.1 & 55 \\
Aceite maiz hidrogenado & 37.5 & 38.0 & 8 & 40.4 & 53 \\
Grasa gallina & - & - & -12 & -0.6 & 47 \\
\hline
\end{tabular}

Las materias primas estudiadas tienen temperaturas de finalización de la fusión ( $T_{\text {endset }}$ ) comprendidas en un rango de apenas $15^{\circ} \mathrm{C}$ (desde $45^{\circ} \mathrm{C}$ hasta $60^{\circ} \mathrm{C}$ ). Esto significa que todas estarán totalmente fundidas a la temperatura de ingesta del caldo. Sin embargo, las temperaturas de comienzo de la fusión ( $T_{\text {onset }}$ ) se encuentran dentro de un rango muy amplio de valores, ya que van desde $-12^{\circ} \mathrm{C}$ (grasa de gallina) hasta $26^{\circ} \mathrm{C}$ (grasa vacuna). Esto implica que dichas materias primas presentan comportamientos muy diferentes desde el punto de vista de su resistencia a las altas temperaturas. Todas (excepto la grasa de gallina) tienen $\mathrm{T}_{\text {onset }}$ del mismo orden o superior a las temperaturas ambientes más frecuentes durante el año en el Uruguay (excepto en los días más tórridos del verano). Por lo tanto, en general, los caldos que las contengan no presentarán problemas de fusión parcial o ablandamiento. En cambio, la grasa de gallina tiene una $T_{\text {pico }}$ inferior a la temperatura de un refrigerador (que suele ser de $4^{\circ} \mathrm{C}$ ) y su fusión comienza a $-12^{\circ} \mathrm{C}$. En consecuencia, esta materia prima está en gran parte fundida a la temperatura ambiente $y$, por lo tanto, puede ocasionar problemas al formar parte de los caldos. Información más amplia al respecto se obtiene de las curvas del contenido en sólidos en función de la temperatura, calculadas a partir de los termogramas mencionados (Gráfica 1).

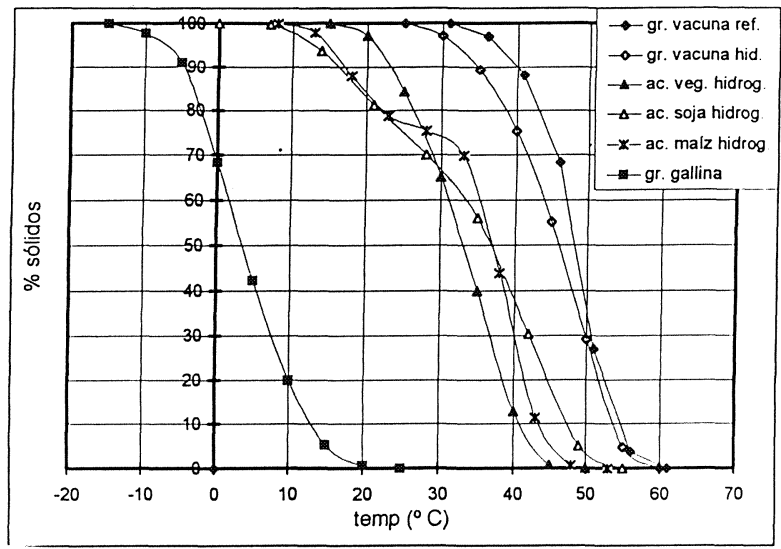

Gráfica 1

Curvas de contenido en sólidos (\%) en función de la temperatura para las materias primas grasas

En la Gráfica 1 se observa que las distintas materias grasas usadas para la elaboración de caldos presentan perfiles térmicos variados, desde la grasa vacuna, más "dura" que las demás a cualquier temperatura, hasta la grasa de gallina, más «blanda» que las demás a cualquier temperatura, pasando por los aceites vegetales parcialmente hidrogenados, de comportamiento intermedio (lo cual se espera de los datos de la Tabla I).

La grasa de gallina es muy particular ya que presenta una fracción líquida importante (hasta un 30\%) aún por debajo de $0^{\circ} \mathrm{C}$; esto es concordante con su 
baja $T_{\text {onset. }}$ (Debido a esto, no se pudo determinar sus puntos de fusión). También presenta una pequeña fracción, no considerada en la curva, que funde por encima de $40^{\circ} \mathrm{C}$ y que es responsable de que su $\mathrm{T}_{\text {endset }}$ sea de $47^{\circ} \mathrm{C}$. Este comportamiento térmico es inadecuado y causa problemas durante el almacenamiento de los cubitos de caldo.

En conclusión, las curvas representadas en la Gráfica 1 se pueden agrupar en tres tipos: a) las dos grasas vacunas; b) los aceites vegetales parcialmente hidrogenados; c) la grasa de gallina. Las dos materias primas más «duras» son las grasas vacunas, tanto la simplemente refinada, como la parcialmente hidrogenada. Ambas presentan curvas casi coincidentes, de las que es importante recalcar el contenido de líquido casi nulo hasta temperaturas del orden de los $35^{\circ} \mathrm{C}$. Ambos materiales se encuentran totalmente sólidos a temperatura ambiente (aún en los días no demasiado cálidos del verano uruguayo), funden con dificultad a la temperatura de la boca pero se encuentran totalmente líquidos a la temperatura de la ingesta del caldo. Estas características están de acuerdo con sus $T_{\text {pico }}$ y sus $T_{\text {endset }}$ respectivas, que son prácticamente iguales (Tabla 1). Esto las convierte en materias primas muy apropiadas para su empleo en cubitos de caldo.

Los tres aceites vegetales parcialmente hidrogenados exhiben curvas similares, si bien son mucho más «blandos» que las grasas vacunas (Gráfica 1), tal como se concluyó de sus $T_{\text {onset, }} T_{\text {pico }} y$ $\mathrm{T}_{\text {endset }}$ (Tabla I). Alrededor de los $50^{\circ} \mathrm{C}$, los tres tienen un contenido de sólidos prácticamente nulo, aunque su comportamiento a temperaturas inferiores a los $40^{\circ} \mathrm{C}$ es algo diferente. El aceite de maíz presenta una mayor constancia del contenido de sólidos en el rango de temperaturas vinculadas con el almacenamiento de los cubitos de caldo (menos de $35^{\circ} \mathrm{C}$ ). El aceite de soja presenta mayor variación en el comportamiento térmico ya que a $35^{\circ} \mathrm{C}$ contiene solamente un $55 \%$ de sólidos y a $20^{\circ} \mathrm{C}$, un $80 \%$. Para mejorar sus propiedades, estos tres materiales podrían ser sometidos a procesos de fraccionamiento térmico controlado para obtener productos más «duros» que los originales o ser hidrogenados más profundamente.

En la Tabla II se resume el contenido en los ácidos grasos principales de las materias primas estudiadas, determinado por GC de los respectivos ésteres metílicos. Para el Uruguay, existen algunos trabajos publicados sobre la composición de las grasas vacunas (Grompone, 1983; Grompone, 1989; Grompone, 1991), de los aceites hidrogenados (Grompone, 1992); y del aceite de pollo (Grompone, 1994).

El contenido en ácido linoleico de la grasa de gallina es muy alto (24.2\%), comparado con el de las otras materias primas; también lo es su contenido en ácido oleico (la suma de ambos es del orden de $65 \%$ ). Esto explica su alto porcentaje de líquido a temperaturas bajas (Gráfica 1).
Tabla II

Composición de las materias primas grasas expresada como porcentaje de los ácidos grasos principales

\begin{tabular}{lcccc}
\hline \multicolumn{1}{c}{ Muestra } & $16: 0$ & $18: 0$ & $18: 1$ & $18: 2$ \\
\hline Grasa vacuna refinada & 25.2 & 29.1 & 32.1 & 2.0 \\
Grasa vacuna hidrogenada & 24.6 & 34.3 & 28.4 & 1.1 \\
Aceite vegetal hidrogenado & 18.6 & 13.2 & 63.4 & 1.3 \\
Aceite soya hidrogenado & 22.2 & 21.2 & 51.3 & 3.0 \\
Aceite maíz hidrogenado & 11.1 & 11.8 & 66.9 & 9.0 \\
Grasa gallina & 19.4 & 7.5 & 40.7 & 24.2 \\
\hline
\end{tabular}

Los aceites vegetales parcialmente hidrogenados presentan un contenido en ácido oleico considerablemente mayor que el de las dos grasas vacunas analizadas; éstas, a su vez, presentan un contenido en ácidos grasos saturados (palmítico y esteárico) mayor que el de los aceites vegetales hidrogenados. Como es de esperar, la grasa vacuna hidrogenada contiene menos ácidos grasos insaturados que la sin hidrogenar, si bien la pequeña diferencia encontrada indicaría un proceso de hidrogenación suave. Estas composiciones justifican los comportamientos témicos diferentes de ambos grupos de materias primas.

Llama la atención el contenido en ácido palmítico del aceite de soja hidrogenado (22.2\%), que no corresponde a este tipo de aceite (el cual suele estar en el orden del $11 \%)$. Aparentemente esta materia prima no sería pura sino que estaría mezclada con otros aceites vegetales también parcialmente hidrogenados (posiblemente algodón, que suele contener un $23 \%$ de ácido palmítico).

\subsection{Caldos}

En la Tabla III se resumen los datos de los puntos de fusión, $T_{\text {onset }}, T_{\text {pico }}$ y $T_{\text {endset }}$ de las mezclas de grasas ya preparadas por el fabricante para la elaboración de los caldos. También se informa los valores correspondientes para las materias grasas extraídas de los caldos de verduras y de gallina ya elaborados (los que fueron confeccionados por el fabricante con mezclas de materias primas similares a las analizadas).

\section{Tabla III}

Puntos de fusión en capilar abierto y en capilar cerrado, temperatura «onset», temperatura de pico y temperatura "endset» del termograma de las mezclas de materias primas grasas y de los extractos lipídicos de los caldos (datos en ${ }^{\circ} \mathrm{C}$ )

\begin{tabular}{lccccc}
\hline \multicolumn{1}{c}{ Muestra } & PF(ca) & PF(cc) & Tonset $_{1}$ & $T_{\text {pico }}$ & $T_{\text {endset }}$ \\
\hline Mezcla caldo gallina & 48.8 & 50.6 & -12 & 50.7 & 59 \\
Mezcla caldo verdura & 50.2 & - & 20 & 51.7 & 62 \\
Extracto caldo gallina & 48.8 & 50.4 & 32 & 49.0 & 56 \\
Extracto caldo verdura & 47.8 & 48.0 & 30 & 50.2 & 58 \\
\hline
\end{tabular}


Se observa que, al igual que las materias primas puras, todas estas muestras presentan $\mathrm{T}_{\text {pico }}$ ligeramente mayores que los puntos de fusión determinados.

Se encuentra también que la mezcla para caldo de verdura y el extracto graso de un caldo de verdura en cubitos (que no necesariamente pertenecen a la misma operación de fabricación) tienen valores concordantes para los puntos de fusión y para las $T_{\text {onset, }}, T_{\text {pico }}$ y $T_{\text {endset. }}$ Lo mismo sucede con la mezcla de grasas para el caldo de gallina y la extraída del correspondiente cubito. Pero si éstas se comparan con la de gallina pura (Tabla I), los valores no concuerdan, debido a que el fabricante empleó una cantidad importante de otras materias primas más "duras» para mejorar su comportamiento térmico inadecuado (lo cual se deberá ver reflejado también en su composición en ácidos grasos). En los termogramas tanto de la mezcla como del extracto del caldo de gallina, se observa un pequeño pico cerca de los $10^{\circ} \mathrm{C}$ (no informado en la Tabla III), que indica la presencia de la grasa de gallina.

A modo de ejemplo ilustrativo, en las Gráficas 2 y 3 se presentan los termogramas de algunas materias primas, superpuestos a los de las mezclas y a los de los extractos de ambos caldos.

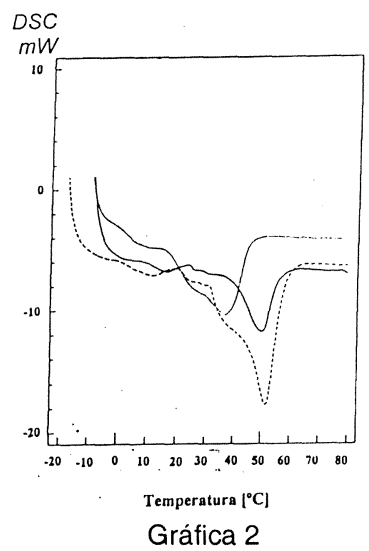

Termogramas del extracto del caldo de verduras (-), de la mezcla de grasas para dicho caldo (- - -), y de aceite vegetal hidrogenado (. . .)

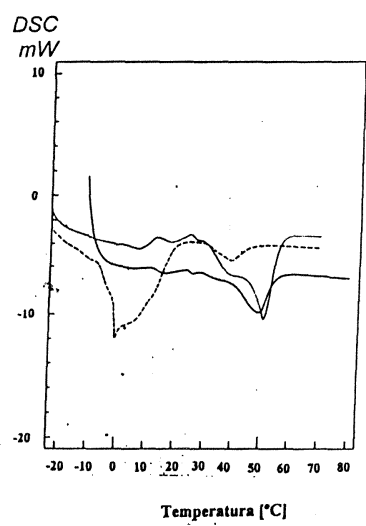

Gráfica 3

Termogramas del extracto del caldo de gallina (-), de la mezcla de grasas para dicho caldo $(. .$.$) , y de la grasa$ de gallina (- - )

\subsubsection{Caldo de verduras}

La Gráfica 4 representa la variación del porcentaje de sólidos en función de la temperatura para la mezcla de grasas empleada en el caldo de verduras, para el extracto lipídico de un cubito de este caldo (que no necesariamente pertenece a la misma operación de fabricación), y para la grasa vacuna refinada.

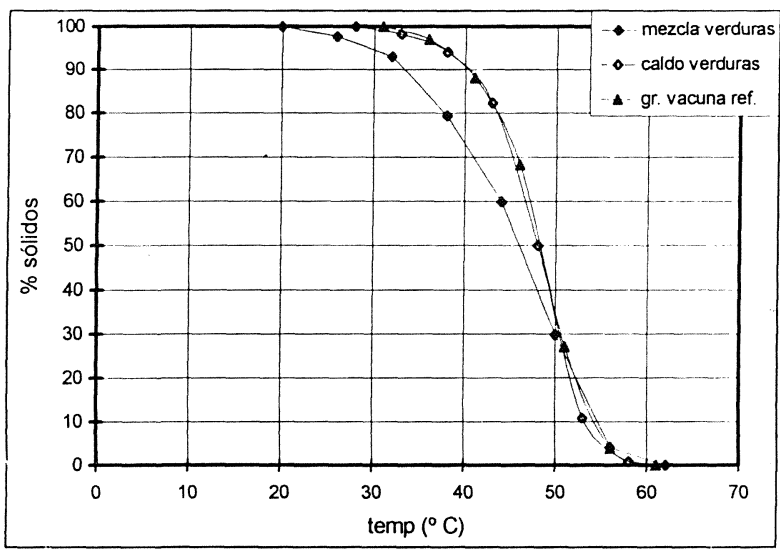

Gráfica 4

Curvas de contenido en sólidos (\%) en función de la temperatura para la grasa vacuna refinada, la mezcla de grasas para el caldo de verduras, y el extracto lipídico de cubito de caldo de verduras

Si bien las curvas de la mezcla de grasas y del extracto del caldo no coinciden, su comportamiento es muy similar. Es interesante notar que la cantidad de líquido recién se hace importante a temperaturas por encima de los $40^{\circ} \mathrm{C}$, lo cual asegura un comportamiento adecuado durante el almacenamiento, aún en verano.

Llama la atención la total coincidencia de la curva de la grasa vacuna con la del extracto lipídico del caldo de verduras, lo cual parece indicar que fue ésta la principal materia prima utilizada. Esto es interesante porque, generalmente, se trata de evitar el uso de grasas de origen animal en los alimentos vegetales ya que el consumidor suele elegirlos cuando quiere mantener una dieta baja en colesterol y en grasas saturadas.

\subsubsection{Caldo de gallina}

La Gráfica 5 representa la curva del porcentaje de sólidos en función de la temperatura para la mezcla de grasas empleada en el caldo de gallina, para el extracto lipídico de un cubito de este caldo (que no necesariamente pertenece a la misma operación de fabricación), y para dos posibles materias primas empleadas: grasa de gallina y grasa vacuna refinada. 


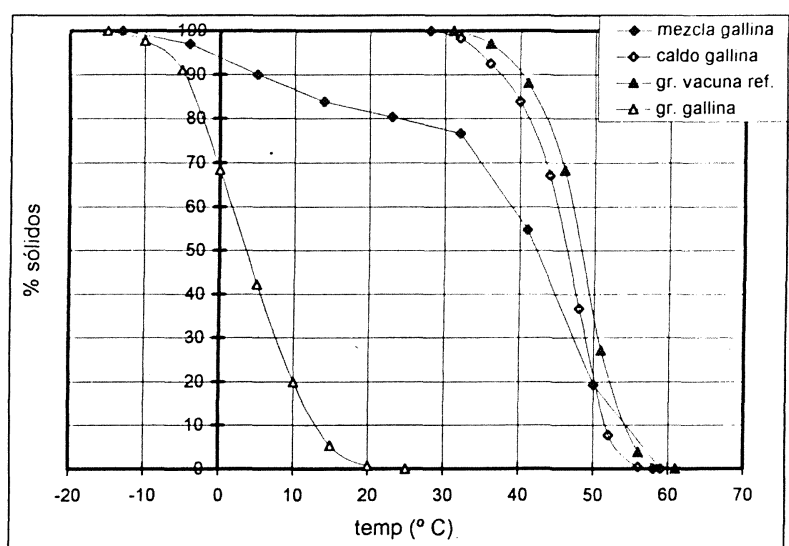

Gráfica 5

Curvas de contenido en sólidos (\%) en función de la temperatura para la grasa de gallina, la grasa vacuna refinada, la mezcla de materias primas grasas para el caldo de gallina, y el extracto lipídico del cubito de caldo de gallina

Las curvas de la mezcla de grasas y del extracto no coinciden, siendo el extracto lipídico del cubito más "duro" que la mezcla, durante casi todo el rango de temperaturas. Esto se puede deber a variaciones en la preparación de las mezclas empleadas en la elaboración de los caldos. Teniendo en cuenta que la grasa de gallina presenta una curva muy baja respecto a las de las otras posibles materias primas, variaciones pequeñas en la cantidad agregada de ella a la mezcla de grasas (además de que la propia gallina aporta su grasa en proporción relativamente variable, en función de su grado de engorde y de la cantidad de carne del ave que se cocinó para preparar el caldo), pueden influir mucho, sobre todo, en la zona de temperaturas bajas de los termogramas. Por otra parte, la composición de la grasa de gallina utilizada como materia prima ( $y$ en consecuencia, su comportamiento térmico) varía considerablemente en función de la alimentación que recibió el animal (Grompone, 1994). En consecuencia, la grasa de gallina (y la propia carne de las gallinas) proveniente de diferentes proveedores, puede tener composición y propiedades muy distintas por lo que ésta es una materia prima que está sujeta a variaciones aleatorias; no así las otras materias primas que tienen una composición más constante, ya sea porque los vacunos no modifican sensiblemente la composición de sus depósitos adiposos en función de su dieta, ya sea porque la composición de los aceites hidrogenados dependen casi exclusivamente del proceso de hidrogenación llevado a cabo por su fabricante.

La grasa de gallina pura es considerablemente más "blanda» que el extracto lipídico del caldo de gallina y éste, a su vez, que la grasa vacuna refinada. Estos dos últimos, sin embargo, tienen curvas muy próximas, aunque son sensiblemente más "duros" que la mezcla de grasas del caldo de gallina. Por lo tanto, cuanto mayor sea el contenido de grasa de gallina del cubito, peor será su comportamiento en los días cálidos (lo que confirma lo dicho anteriormente respecto a los datos de la Tabla III). Obviamente el caldo de gallina tiene agregados muy importantes de otras grasas más "duras" que la de gallina para mejorar el comportamiento térmico de ésta (puede ser tanto grasa vacuna como alguna de las otras materias primas hidrogenadas). La relación entre las grasas "duras" agregadas y la de gallina debe ser muy alta dado que la curva del contenido en sólidos en función de la temperatura para la mezcla final es muy cercana a aquéllas y muy alejada de ésta. Sin embargo, aún en las mezclas como la correspondiente al cubito de caldo, no se puede eliminar totalmente el efecto de los componentes que funden a temperaturas muy bajas y que son aportados por la grasa de gallina (que como ya se dijo, se refleja en el termograma como un pequeño pico a temperaturas por debajo de $10^{\circ} \mathrm{C}$ ). Estos componentes serían responsables del mayor ablandamiento del cubito de gallina respecto al de verdura, constatado aún a temperatura ambiente. Esta variabilidad en el comportamiento térmico de las mezclas de grasas para caldo de gallina, debida a su composición no constante, hace que el almacenamiento en verano de este tipo de caldos sea muy problemático porque se corre el riesgo de que los confeccionados con tachadas demasiado "blandas» exuden aceite líquido apenas aumente un poco la temperatura ambiente.

\subsubsection{Comparación entre los caldos de verduras y de gallina}

Si se compara la curva del porcentaje de sólidos del extracto lipídico del caldo de verdura (gráfica 4) con la del extracto del caldo de gallina (gráfica 5) se encuentra una similitud significativa, aunque el de gallina es ligeramente más «blando» a todas las temperaturas. Ello indicaría que se usaron materias primas similares para los dos tipos de caldos aunque es de tener en cuenta la variabilidad ya comentada para el de gallina. En ese sentido, el caldo de verduras puede tener una composición lipídica más fácilmente controlada puesto que ésta proviene exclusivamente de la mezcla realizada por el fabricante ya que las verduras componentes del caldo no aportan materiales grasos.

En la Tabla IV se resume la composición porcentual en los ácidos grasos principales para las mezclas de grasas y para los extractos de los caldos. 
Tabla IV

Composición expresada en porcentaje de los ácidos grasos principales de las mezclas de materias primas grasas y de los extractos lipídicos de los caldos de verduras y de gallina en porcentaje de los ácidos grasos principales

\begin{tabular}{lcccc}
\hline \multicolumn{1}{c}{ Muestra } & $16: 0$ & $18: 0$ & $18: 1$ & $18: 2$ \\
\hline Mezcla gallina & 23.8 & 27.1 & 32.5 & 6.1 \\
Mezcla verdura & 25.1 & 31.4 & 29.3 & 3.8 \\
Extracto caldo gallina & 23.1 & 23.7 & 35.1 & 7.4 \\
Extracto caldo verdura & 24.1 & 28.1 & 32.7 & 4.8 \\
\hline
\end{tabular}

Se observa una similitud pronunciada entre la composición de la mezcla y la del caldo correspondiente. También es interesante la similitud entre la composición de ambos tipos de caldo. De los cromatogramas (omitidos en este trabajo por razones de brevedad) surge como evidente la presencia de grasa vacuna en ambos tipos de caldo (esto confirma lo que parecía surgir de la Gráfica 4 para el caldo de verduras). Este es un dato importante desde el punto de vista nutricional, porque el consumidor no suele estar advertido al respecto y presupone que los caldos de verdura no contienen grasas de origen animal.

Comparando tanto las mezclas de grasas de ambos tipos de caldo como los correspondientes extractos de los cubitos, se nota que los de gallina contienen un poco menos de ácidos grasos saturados y un poco más de insaturados, lo que justifica sus puntos de fusión menores (Tabla III). EI mayor contenido en ácido linoleico del caldo de gallina frente al de verdura se justifica por el aporte importante que de éste hace la grasa de gallina

\section{CONCLUSIONES}

Para un alimento con las características propias de los cubos de caldo deshidratado, son necesarias materias primas grasas cuyas propiedades térmicas sean adecuadas para las condiciones ambientales en las cuales se almacenará. En ese sentido, la calorimetría diferencial de barrido es una herramienta que aporta información muy valiosa. Algunas de las materias primas estudiadas (como la grasa vacuna hidrogenada) se pueden considerar apropiadas para la confección de caldos en cubitos. Como en los caldos de gallina, además de la grasa de gallina agregada, siempre existirá el aporte de grasa de la propia carne de ave, se debe adicionar otras grasas para «endurecer» el producto final.

Para algunas materias primas, en especial la grasa de gallina, parece conveniente modificar el contenido en fracciones de bajo punto de fusión, de modo de mejorar su comportamiento térmico. Una solución se podría encontrar en el fraccionamiento térmico controlado o en una hidrogenación adicional para lograr fracciones más "duras», que lograran mantener los caracteres organolépticos sin la influencia negativa en la estabilidad frente a la variación de temperatura.

\section{BIBLIOGRAFIA}

Al-Rashood, K.; Abou-Shaaban, R. R. A; Abdel-Moety, E. M. and Rauf, A. (1996).—-Compositional and Thermal Characterization of Genuine and Randomized Lard: A comparative study".-J. Amer. Oil Chem. Soc. 73, (3), 303-309.

Amer, M. A.; Kupranycz, D. B. and Baker, B. E. (1985)._ «Physical and Chemical Characteristics of Butterfat Fractions Obtained by Crystallization from Molten Fat».J. Amer. Oil Chem. Soc. 62, (11), 1551-1557.

AOCS (1993).- "Official Methods and Recommended Practices of the American Oil Chemists'Society".-4th Edition (1989, revisión 1993).--Firestone, D. (Editor), Champaign.

Bornaz, S.; Fanni, J. and Parmentier, M. (1994)._- «Limit of the Solid Fat Content Modification of Butter».-J. Amer. Oil Chem. Soc. 71 (12), 1373-1380.

Borwankar, R. P.; Frye, L. A.; Blaurock, A. E. and Sasevich, F. J. (1992). - «Rheological Characterization of Melting of Margarines and Tablespreads".-J.Food Eng. 16, 55-74

Dimick, P. S.; Reddy, S. Y. and Ziegler, G. R. (1996).— «Chemical and Thermal Characteristics of Milk-Fat Fractions Isolated by a Melt Crystallization».- $\mathrm{J}$. Amer. Oil Chem. Soc. 73, (12), 1647-1652.

Dubinsky, E. (1995).- «Productos hidrogenados: producción y especificaciones".-Aceites y Grasas 5, (19), 192-203.

Fouad, F. M.; van de Voort, F. R.; Marshall, W. D. and Farrell, P. G. (1990).— «A Critical Evaluation of Thermal Fractionation of Butter Oil».-J. Amer. Oil Chem. Soc. 67, (12), 981-988.

Garti, N. and Sato, K. (1988).- -Crystallization and polymorphism of fats and fatty acids".-Marcel Dekker Inc, New York.

Grompone, M. A. and Moyna, P. (1983).- «Characteristics of Uruguayan beef tallow».-J. Amer. Oil Chem. Soc. 60, (7), 1331-1332

Grompone, M. A. (1989).— «Physicochemical properties of fractionated beef tallow».-J. Amer. Oil Chem. Soc. 66, (2), 253-255.

Grompone, M. A. (1991).- «Los sebos uruguayos y su empleo en la industria nacional».--Rev. Ing. Quim. 3, (1), 14-18.

Grompone, M.A. (1992).- «Propiedades de los aceites vegetales modificados por hidrogenación, interesterificación y fraccionamiento".-Grasas y Aceites 43 (6), 330-335.

Grompone, M.A.; Guerra, J.F.; Pazos, N.A.; Méndez, E.; Lucas, E.; Jachmanián, I. y Collazzi, P. (1994)."Fraccionamiento térmico de aceite de pollo".Grasas y Aceites, 45, (6), 390-394.

Kapseu, C. and Kayem, G.J. (1994)._-Analysis of cottonseed oil and its fractions by differential scanning calorimetry".-J.Food Eng. 21, 225-234.

Man, Y.B. Che and Swe, P.Z. (1995).- «Thermal analysis of failed-batch palm oil by differential scanning calorimetry". - J. Amer. Oil Chem. Soc. 72 (12), 1529-1532.

Zeitoun, M. A. M.; Neff, W.E. ; List, G.R. and Mounts, T.L. (1993)._- «Physical properties of interesterified fat blends".-J. Amer. Oil Chem. Soc. 70 (5), 467-471.

Recibido: Enero 1998 Aceptado: Mayo 1998 